\title{
Numerical Simulations of Cardiac Tissue Excitation and Pacing Using the Bidomain Model
}

\author{
Bradley J. Roth* \\ Department of Physics, Oakland University, Rochester, MI 48309, USA
}

\begin{abstract}
The last two decades have produced significant advances in our theoretical understanding of cardiac pacing. The bidomain model, a mathematical representation of the anisotropic electrical properties of cardiac tissue, has provided insight into many long-standing mysteries of pacing, such as the mechanisms of make and break excitation, the shape of the strength-interval curve, and the induction of reentry by burst pacing. This paper reviews the application of the bidomain model to these and other topics, and summarizes our theoretical understanding of how electrical excitation of the heart works.
\end{abstract}

Keywords: Pacing, bidomain, mathematical modeling, make excitation, break excitation, cathode, anode, strength-interval curve, burst pacing.

\section{INTRODUCTION}

Last year the cardiac electrophysiology community celebrated the $50^{\text {th }}$ anniversary of the first permanent implantation of a pacemaker in the United States $[1,2]$. Despite this long history, our understanding of how pacemakers influence cardiac tissue is still developing, and many important questions have only recently been answered [3, 4]. For instance, how does pacemaker current redistribute between the intracellular and extracellular spaces to induce a transmembrane potential? Can the threshold stimulus strength be predicted from basic theory? How does "make" excitation differ from "break" excitation? Can cardiac tissue be excited by an anode? What is the mechanism for the dip in the anodal strength-interval curve? How does burst pacing lead to arrhythmias? I believe these questions are critically important for two reasons. First, answers would help us to gain a better understanding of how pacemakers work, and perhaps thereby allow us to build better pacemakers. Second, pacing provides a simple test bed to evaluate our models of how electric fields influence the electrical properties of the heart. If we can predict accurately what happens during pacing, we will be more confident that we can apply the same models to defibrillation [5].

The bidomain model describes the interaction of electric current with cardiac tissue [6-8]. This two- or threedimensional cable model represents the electrical properties of the intracellular and extracellular spaces each as macroscopic anisotropic continua, coupled through the cell membrane Fig. (1). The bidomain model was developed in the late 1970's [9-13], and was applied to more complex problems in cardiac electrophysiology in the 1980s [14-19].

*Address correspondence to this author at the Department of Physics, Oakland University, Rochester, USA, MI 48309; Tel: 248 370-4871; Fax: 248 370-3408; E-mail: roth@oakland.edu
This work set the stage for the use of the bidomain model to study cardiac pacing.

\section{UNIPOLAR STIMULATION}

The first application of the bidomain model to pacing was by Sepulveda et al. in 1989 [20]. They considered a unipolar cathode injecting current into the extracellular space, with a distant anode. Fig. (2) shows the resulting transmembrane potential produced around the cathode. The location of the cathode is indicated by the dot, the myocardial fibers are oriented along the $X$ axis, the stimulus duration is long enough that the transmembrane potential has reached steady state, and the tissue is passive; the model does not include the opening and closing of ion channels. Under the cathode the tissue is depolarized (positive). Depolarization contours a few millimeters from the cathode have a "dog-bone" shape, extending farther in the direction perpendicular to the electrodes than parallel to them. However, about $2 \mathrm{~mm}$ from the cathode in the direction along the fibers is a region of hyperpolarization (negative), called a "virtual anode" because the negative transmembrane potential is not associated with a nearby anodal electrode. Only one quarter of the tissue sheet is shown in Fig. (2), so two virtual anodes exist, one on each side of the cathode.

A corollary of this prediction is that an anode causes depolarization at two locations along the fiber direction at either side of the stimulating electrode, providing a mechanism for anodal excitation. In this case, the wave front originates from the "virtual cathodes." Understanding anodal stimulation is important, because it may be advantageous to stimulate with an anode in certain situations [21-24].

Roth and Wikswo [25] generalized the Sepulveda et al. calculation by extending it to a three-dimensional volume of tissue and adding a model for the sodium channel kinetics, allowing them to simulate the excitation of action potential 

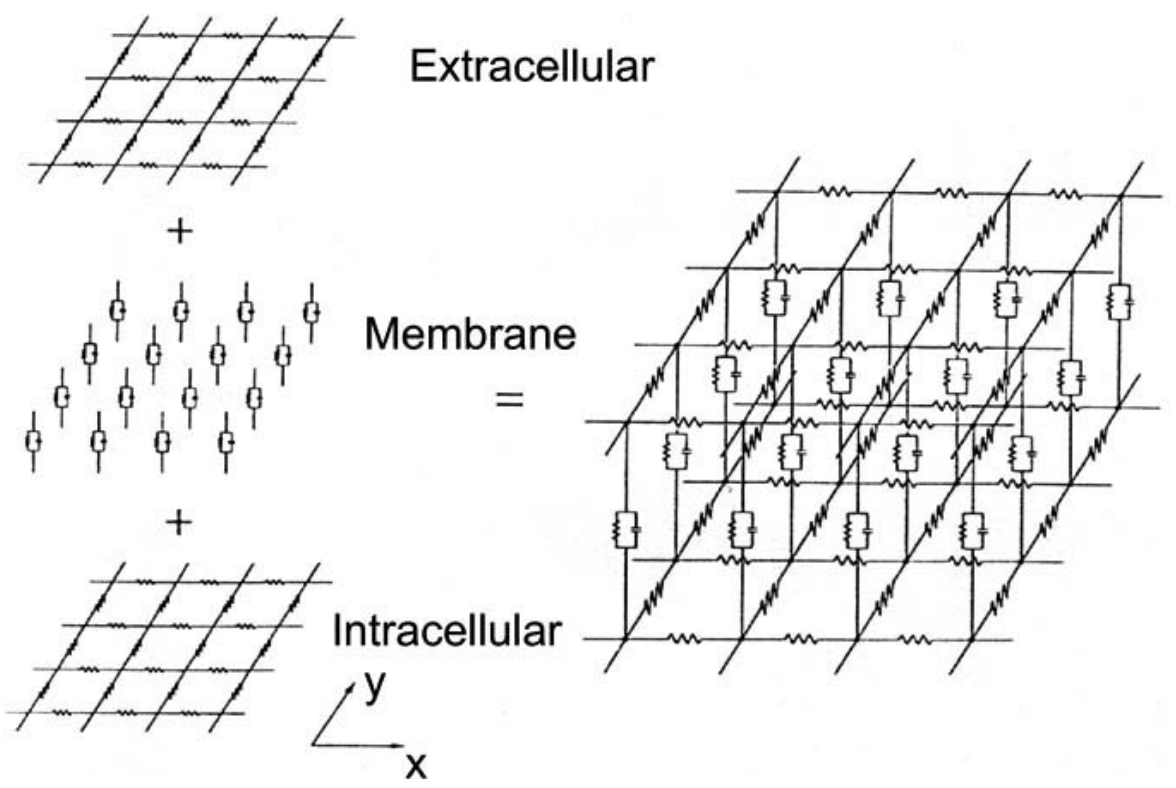

Fig. (1). A schematic diagram of the bidomain model, which represents the anisotropic electrical properties of the intracellular and extracellular spaces. In this passive model, the membrane is represented by a capacitor and single resistor. Modified from [33].

wave fronts. They found that excitation arises from the depolarized region of tissue, and occurs for both cathodal and anodal stimulation. Muzikant and Henriquez [26-28] extended this model to include the rotating fiber direction across the heart wall. For large electrodes, the distribution of depolarization and hyperpolarization is modified [29], with strongest depolarization under the edge of the cathode [30].

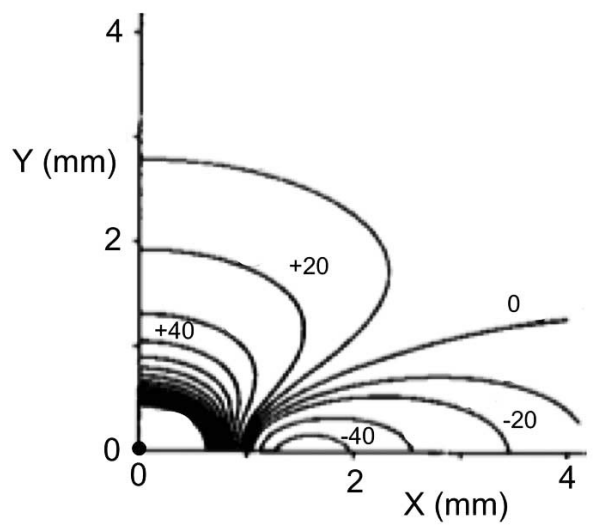

Fig. (2). The steady-state transmembrane potential induced in a two-dimensional sheet of tissue by a unipolar extracellular electrode, located at the origin (dot). Only one quarter of the sheet is shown. The fibers lie along the $\mathrm{X}$ direction. Contours are drawn every $10 \mathrm{mV}$. The many closely spaced contours near the electrode are not shown. Modified from [20].

In an alternative and more theoretical approach, perturbation theory applied to the passive bidomain model provides analytical equations that predict the distribution of virtual cathodes and anodes [31, 32], which are qualitatively consistent with the numerical stimulations $[20,25]$. These calculations highlight the condition that "virtual electrodes" result from the unequal anisotropy ratios in the intracellular and extracellular spaces [33]. The ratio of conductivities parallel and perpendicular to the myocardial fibers is about 10 in the intracellular space, and is approximately 2.5 in the extracellular space [34]. For those preferring an intuitive rather than mathematical explanation of why virtual electrodes appear during unipolar stimulation, the phenomenon can be explained using qualitative arguments related to how the anisotropy of the electrical conductivity rotates the current density vectors differently in the intracellular and extracellular spaces [35].

Although the presence of virtual anodes during cathodal unipolar stimulation is surprising and counter-intuitive, one should remember that these virtual anodes play an important role in wave front dynamics primarily for relatively strong stimuli. When Aker et al. [36] used subthreshold stimuli to determine the length constant of cardiac tissue, they did not observe any signs of virtual cathodes or anodes, nor did they record dog-bone shaped contours of transmembrane potential. However, when they increased the stimulus strength to twenty times diastolic threshold, the bidomain effects predicted by Sepulveda et al. [20] were obvious. Virtual electrodes play a particularly important role during the response of the heart to strong electrical shocks, such as during defibrillation [37-39].

The theoretical predictions of the bidomain model were first tested by Wikswo et al. [40], who used extracellular electrodes to measure wave front arrival times in a dog heart following unipolar stimulation. They determined that the wave fronts originated farther from the cathode perpendicular to the fibers than parallel to them, consistent with Sepulveda et al.'s [20] prediction. However, a direct measurement of the transmembrane potential produced by a cathode had to wait for the application of optical mapping techniques to cardiac electrophysiology in the mid 1990s, when three groups almost simultaneously published data that confirmed qualitatively the theoretical prediction of adjacent virtual anodes and cathodes during unipolar stimulation [41-43]. 


\section{OPTICAL MAPPING OF TRANSMEMBRANE POTENTIAL}

Optical mapping is one of the most powerful tools in the cardiac electrophysiologist's toolkit [44]. It became widely used for cardiac electrophysiology just as research based on the bidomain model was generating predictions that required experimental verification [45-47]. A fluorescent dye is introduced into tissue and absorbed by the membrane. When light is shined on the tissue, the resulting fluoresced light depends on the transmembrane potential, allowing the optical measurement of electrical behavior. The method has its weaknesses, which make comparing experimental data to theoretical predictions a challenge $[48,49]$. To understand one of the problems, we first analyze the three-dimensional distribution of transmembrane potential. When a unipolar cathode is located in a bath superfusing a slab of cardiac tissue [50], the distribution of transmembrane potential qualitatively resembles the prediction of Sepulveda et al. [20], and decays with depth into the tissue Fig. (3). Yet, under some conditions the transmembrane potential distribution can be more complicated. For instance, under some conditions the virtual anode is buried under the tissue surface [51]. When examining bipolar stimulation [52], Latimer and Roth [50] predicted such buried hyperpolarization Fig. (4), arising because of the boundary conditions applied at the tissue-bath interface [53, 54]. The very existence of the virtual anode would not be evident from measurements of transmembrane potential at the tissue surface. Buried hyperpolarization may play an important role in defibrillation by creating hidden "tunnels" of excitable tissue [55].

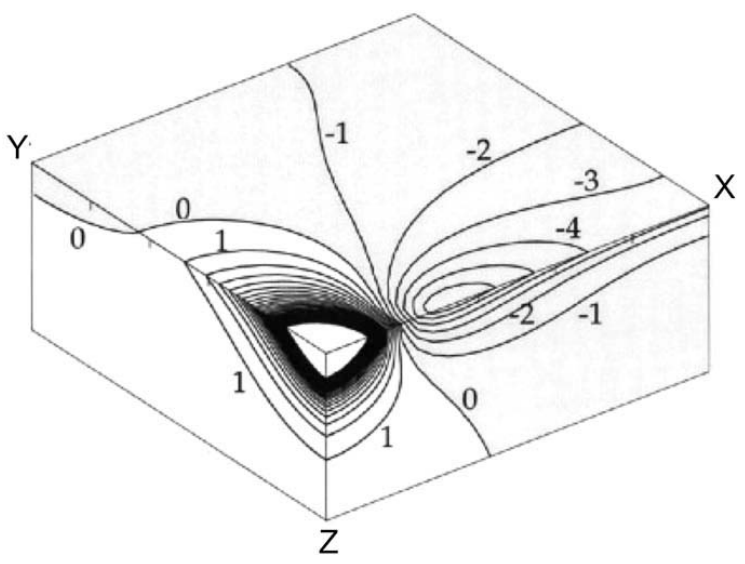

Fig. (3). The steady-state transmembrane potential produced in cardiac tissue when a cathode carrying a current of $1 \mathrm{~mA}$ is $0.2 \mathrm{~mm}$ above the origin in the bath superfusing the tissue. Contours are drawn every $\mathrm{mV}$, and tick marks correspond to $1 \mathrm{~mm}$. The fibers are parallel to the $\mathrm{X}$ axis, and the plane $\mathrm{Z}=0$ corresponds to the tissue-bath surface. The many closely spaced contours near the electrode are not shown. Modified from [50].

But do optical recordings measure the transmembrane potential at the surface? Several experimental measurements have called this assumption into doubt [41, 56-59]. Both the optical penetration of light and the transmembrane potential fall off with depth into the tissue. When the decay of light occurs with a longer space constant than the decay of potential, the optical signal is a distorted representation of the

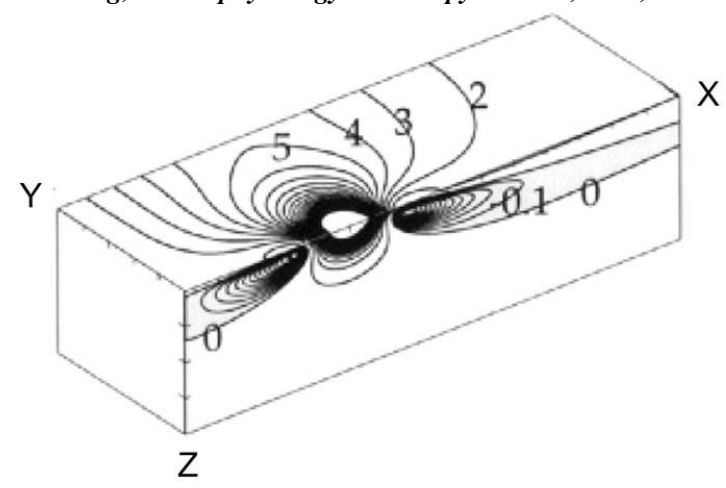

Fig. (4). The steady-state transmembrane potential produced in cardiac tissue during bipolar stimulation. A cathode carrying a current of $1 \mathrm{~mA}$ is $0.2 \mathrm{~mm}$ above the tissue in the bath superfusing the tissue. An anode is symmetrically placed at negative $\mathrm{X}$ values. Contours are drawn every $\mathrm{mV}$, and tick marks correspond to $1 \mathrm{~mm}$. The fibers are parallel to the $\mathrm{X}$ axis and the plane $\mathrm{Z}=0$ corresponds to the tissue-bath surface. The many closely spaced contours near the electrode are not shown. Modified from [50].

surface transmembrane potential [60]. Prior and Roth [61] compared the transmembrane potential at the tissue-bath surface with the predictions of the optically measured transmembrane potential during unipolar stimulation Fig. (5). They found that very close to the electrode the transmembrane potential on the tissue surface falls off rapidly and non-exponentially with distance from the electrode (Fig. (5), dashed curves). However, the predicted optical measurement underestimates the transmembrane potential near the electrode dramatically, and falls off exponentially with distance from the electrode. These simulations imply that the actual transmembrane potential very near an electrode may be much larger than what is measured optically, and suggest that length constant measurements based on the assumption of exponential behavior of the transmembrane potential may,
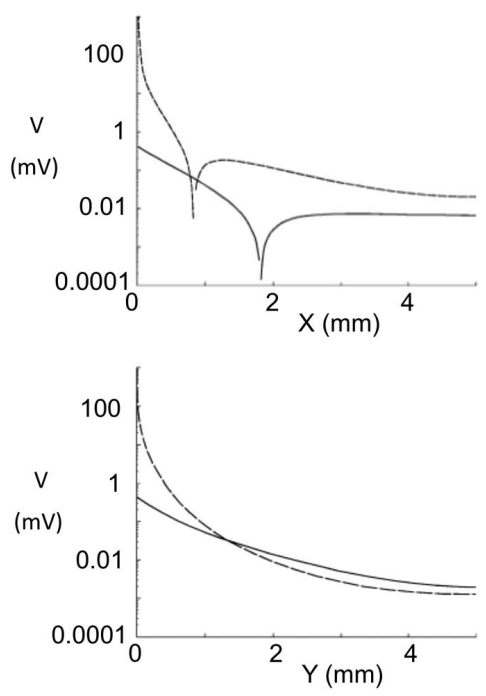

Fig. (5). The absolute value of the steady-state transmembrane potential $\mathrm{V}$ produced by unipolar stimuation as a function of: upper panel, distance parallel to the fibers, X; lower panel, distance perpendicular to the fibers, Y. The dashed curves are the surface transmembrane potential, and the solid curves are the calculated optical mapping signals. Modified from [61]. 
in fact, tell us as much about smearing of optical signals as about the electrical properties of cardiac tissue [36, 62]. New methods are being developed to extract the transmembrane potential versus depth from the optical signal, including techniques based on optical mapping using multiple wavelengths of light [63, 64], optrodes [65-67], bulk versus surface staining of fluorescent dyes [68], and transillumination [59].

\section{MAKE AND BREAK EXCITATION}

Long before the bidomain model was developed, cardiac electrophysiologists discovered that four mechanisms existed for excitation of cardiac tissue: cathode make, anode make, cathode break, and anode break [69-72]. Simulations based on the bidomain model helped explain these different mechanisms [73]. Cathode make excitation is the mechanism usually associated with pacing; depolarization under the cathode excites an action potential wave front. The term "make" implies that excitation occurs promptly after turning on the stimulus. Anode make excitation arises from the existence of virtual cathodes during anodal stimulation; the wave front originates a millimeter or two from the anode along the fiber direction. Because strong stimuli are required to produce significant virtual cathodes during anodal stimulation, anode make excitation has a higher threshold than cathode make excitation.

"Break" excitation occurs after the stimulus is turned off, and is often important when stimulating refractory tissue. During a strong cathodal stimulus, the depolarization under the electrode may not excite an action potential because the tissue is refractory, but the hyperpolarization at the virtual anode causes the tissue to recover from refractoriness quickly (de-excite) [5]. At the end of the stimulus pulse, the depolarization interacts electrotonically with the hyperpolarized and now excitable tissue in the virtual anode, initiating a wave front that propagates along the fiber direction (actually two wave fronts, because there are two virtual anodes, one on each side of the cathode). This mechanism requires hyperpolarization at the virtual anode that is strong enough to de-excite the action potential, and therefore cathode break excitation has a higher threshold than cathode make excitation. Finally, anode break excitation occurs in refractory tissue following the end of an anodal stimulus, when depolarization at the virtual cathode interacts electrotonically with the hyperpolarized and now excitable tissue under the anode, exciting a wave front. Anode break excitation has the highest threshold of all, because the depolarization at the virtual cathode must be quite strong to initiate the wave front. Wikswo et al. [43] analyzed these four mechanisms using optical mapping, and found behavior consistent with the bidomain predictions.

Fig. (6) shows the stimulation thresholds for each mechanism, predicted using the bidomain model [73]. The order (cathode make, anode make, cathode break, and anode break) is consistent with experimental observations [70]. The cathode make threshold is very small, and is as low as 0.01 $\mathrm{mA}$. This is smaller than typical pacing thresholds, but such small thresholds have been measured experimentally using small electrodes in carefully controlled conditions [72]. Both anode make and cathode break thresholds are around $1 \mathrm{~mA}$, and anode break threshold is at least a factor of ten higher.
The remaining discrepancies between theory and experiment have been explored in detail [48].

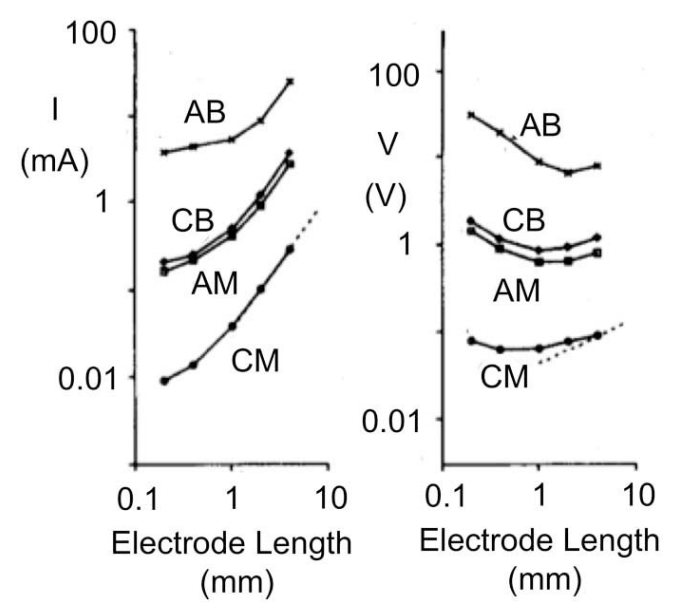

Fig. (6). The rheobase threshold stimulus as a function of electrode length, for cathode make (CM), anode make (AM), cathode break $(\mathrm{CB})$, and anode break (AB) stimulation. Left panel, stimulus current, and right panel, stimulus voltage. Modified from [73].

The anode break mechanism predicted by the bidomain model is quite different than the classical anode break mechanism that Hodgkin and Huxley studied in nerve axons [74]. In nerves, hyperpolarization increases the excitability of the membrane by modifying the state of various ion channels, particularly the sodium channel inactivation gate. Anode break can take place in space-clamped nerves and therefore does not required electrotonic interactions. In cardiac tissue, on the other hand, space clamped models do not predict anode break excitation; electrotonic interaction between adjacent depolarized and hyperpolarized regions is necessary. Some researchers have questioned the validity of the bidomain mechanism for anode break excitation, suggesting it may arise from membrane effects like in nerves. Ranjan et al. [75, 76] suggested that a hyperpolarizationactivated inward current may be responsible for anode break excitation. Simulations that include both bidomain effects and a hyperpolarization-activated current suggest that the two mechanisms could be distinguished by careful examination of the origin of the wave front $[77,78]$. If the bidomain mechanism dominates, excitation should originate at the edge of the virtual anode, adjacent to depolarized tissue. If a membrane current mechanism dominates, excitation should arise where hyperpolarization is strongest, which is usually at the center of the virtual anode. Other researchers have also examined the question of anode break excitation, suggesting that it may arise from electrode effects [79]. Observations of diastolic anode break excitation are difficult to understand using bidomain mechanisms, because the anode make threshold should be lower than the anode break threshold in resting tissue [80]. Nonlinear effects caused by the inward rectifying potassium current also influence the distribution of depolarization and hyperpolarization during pacing [81].

\section{STRENGTH INTERVAL CURVES}

One longstanding puzzle in cardiac electrophysiology is the dip of the anodal strength-interval curve [72, 82-84]. The strength-interval curve measures the threshold strength of a 
second stimulus as a function of the time (or interval) between the first and second stimuli. For a cathodal stimulus, the strength-interval curve decreases as the interval increases, because the tissue is easier to excite as it recovers from refractoriness. However, for an anodal stimulus there is a section of the strength-interval curve that increases as the interval increases, thereby paradoxically making the tissue harder to stimulate as it recovers from refractoriness.

Bidomain simulations have clarified the mechanism of the dip in the strength-interval curve [85]. Fig. (7) shows the predicted strength-interval curves for four different stimulus pulse durations. If we focus on the $5 \mathrm{~ms}$ stimulus, the cathodal curve decreases monotonically with interval, whereas the anodal curve increases between 290 and $300 \mathrm{~ms}$ (the dip). The anodal curve is divided into two sections, corresponding to break excitation for intervals between 290 and $325 \mathrm{~ms}$, and to make excitation for intervals greater than $325 \mathrm{~ms}$, with an abrupt transition between the two. The make section is monotonically decreasing, but the break section contains the dip. To understand why the dip is present, realize that anode break excitation requires a source of depolarization that interacts electrotonically with the hyperpolarized and excitable tissue under the anode. This depolarization can arise from two sources: the virtual cathode adjacent to the anode, or a previous action potential. As the interval decreases, the depolarization arising from the previous action potential increases. For intervals between 290 and $300 \mathrm{~ms}$, this source of depolarization is dominant, so the excitation becomes easier the earlier the stimulus is applied. If the interval is very early (less than $290 \mathrm{~ms}$ in this case), anode break excitation occurs but once the resulting wave front traverses the virtual anode it encounters refractory tissue and fails to propagate. The dip of the anodal strength-interval curve is especially prominent for longer pulse durations. Sidorov et al. [86] studied the strength-interval curves using optical mapping, and found similar behavior as predicted by the bidomain model.

This mechanism for the dip in the anodal strengthinterval curve implies that the dip should occur over the same interval range as the repolarization phase of the previous action potential, at which time the tissue is recovering from refractoriness. One way to test this prediction is to make the repolarization phase of the previous action poten-
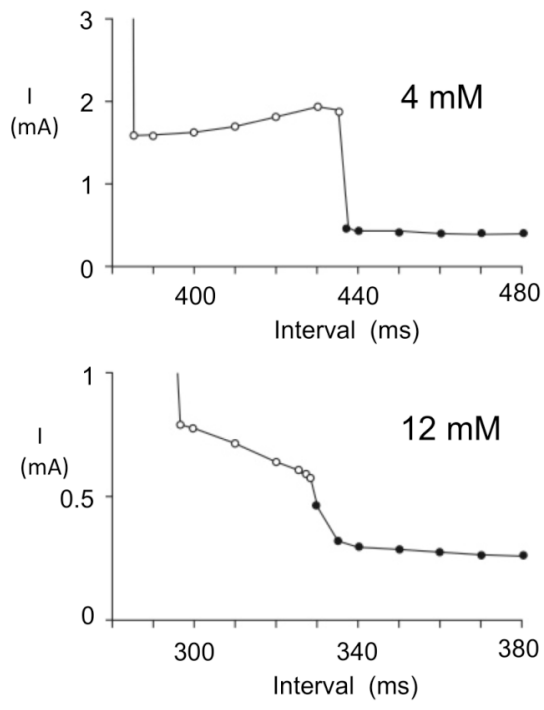

Fig. (8). Anodal strength-interval curves for an extracellular potassium ion concentration of: upper panel, $4 \mathrm{mM}$; and lower panel, 12 $\mathrm{mM}$. Open circles are break excitation, and closed circles are make excitation. The stimulus duration is $20 \mathrm{~ms}$. Modified from [88].

tial occur at a different time than the recovery from refractoriness. This is possible in cases of post-repolarization refractoriness, when the action potential remains refractory long after the previous action potential has returned to rest. One way to produce post-repolarization refractoriness is to raise the extracellular potassium ion concentration. Sidorov et al. [87] studied hyperkalemia using optical mapping, and found that the diastolic anodal excitation mechanism changed from make $(4 \mathrm{mM} \mathrm{KCl})$ to break $(8 \mathrm{KCl})$. Bidomain simulations of the anodal strength-interval curve make similar predictions [88]. Although the diastolic excitation mechanism is make in the simulations, the relative thresholds for break and make excitation decrease as potassium concentration increases. Moreover, at high potassium the dip in the break section of the anodal strength-interval curve disappears. This is caused by the significant post-repolarization refractoriness at high potassium concentration, as shown in Fig. (9). At 4 $\mathrm{mM}$, the make/break transition occurs just as the transmembrane potential from the previous action potential returns to
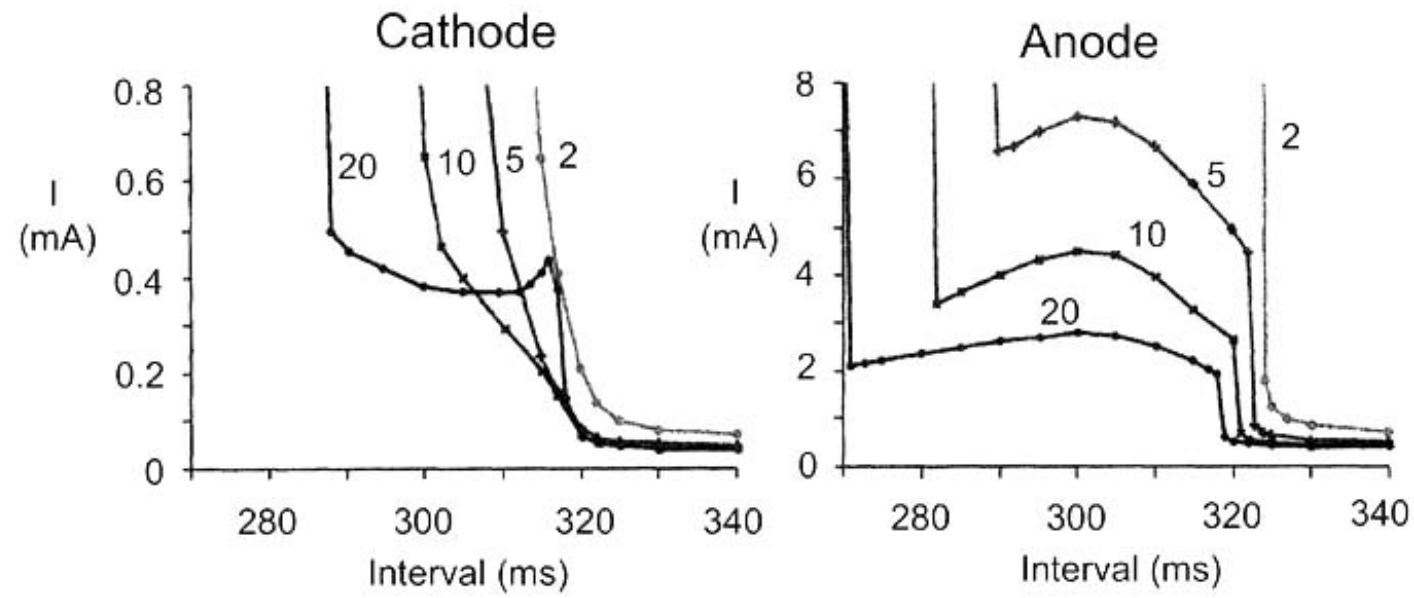

Fig. (7). The strength-interval curve for left panel: a cathodal stimulus; and right panel, anodal stimulus. Four stimulus durations are shown: 2, 5, 10, and $20 \mathrm{~ms}$. Modified from [85]. 
rest. At $12 \mathrm{mM}$, the make/break transition occurs $50 \mathrm{~ms}$ after the action potential has returned to rest.

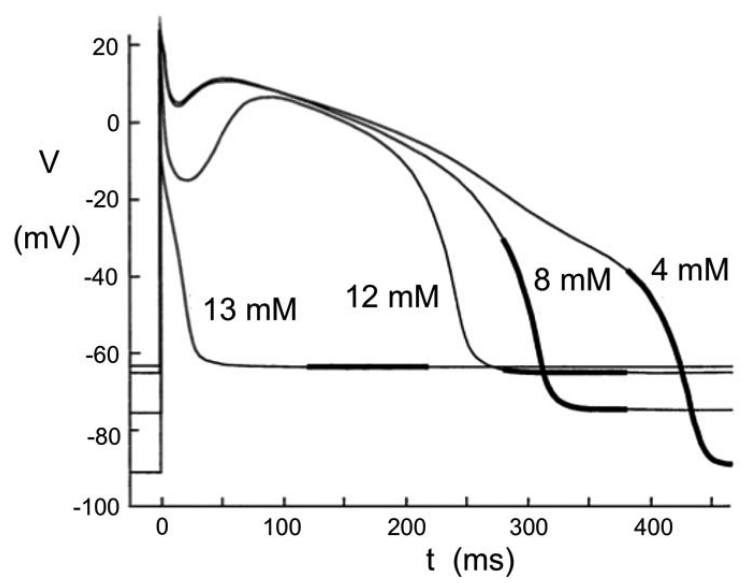

Fig. (9). The transmembrane potential as a function of time, calculated adjacent to the stimulating electrode, for extracellular potassium ion concentrations of $4,8,12$, and $13 \mathrm{mM}$. The thick sections indicate the time for which the strength-interval curve was calculated in Fig. (8). Modified from [88].

Recently, Joung et al. [89] suggested that the intracellular calcium concentration and the sodium-calcium exchange current may play a role in the mechanism of the dip of the anodal strength-interval curve. Simultaneous measurements of transmembrane potential and intracellular calcium concentration $[90,91]$ indicate that virtual electrodes can impact calcium, but the influence of calcium on the excitation threshold remains an open question.

Mehra et al. [92] observed that the strength-interval curves derived from chronically implanted electrodes are time dependent. Initially the refractory period is longer for cathodal compared to anodal stimuli, but weeks after implantation the refractory period becomes shorter for cathodal compared to anodal stimuli. Bidomain stimulations provided a different interpretation of these data [93]. The thresholds for both make and break excitation varies with electrode size. As a region of scar tissue forms around the electrode tip, the electrode size effectively increases, and therefore electrode size is a good analog to scar formation. For small electrodes, both the anodal and cathodal thresholds are low, and anode break excitation is the mechanism that operates at short intervals Fig. (10). For larger electrodes, the anode break threshold becomes so large that the stimulator Mehra et al. [92] used could not provide a large enough stimulus to trigger anode break. In that case, the break section of the anodal strength-interval curve cannot be observed, and the effective "refractory period" is determined by the make section alone Fig. (10). The net effect is to make the anodal refractory period appear to become longer. Of course, this is just an illusion caused by the output limit of the stimulator. A more powerful stimulator would uncover the anode break section of the strength-interval curve, implying a shorter refractory period.

\section{RAPID RATE PACING}

One way to induce fibrillation in the heart is rapid rate, or burst, pacing [94, 95]. Janks and Roth [96] used the bidomain model to simulate a train of stimuli, and found that rapid pacing dramatically reduces the threshold for induction of reentry, from $8.6 \mathrm{~mA}$ for a two-shock (S1-S2) protocol to $0.9 \mathrm{~mA}$ for burst pacing. Fig. (11) summarizes these results. For stimulus trains with a long interval between pulses, the tissue responded in a 1:1 manner and no reentry occurred. For shorter intervals, the stimuli arrived so rapidly that the tissue could only be excited by every other stimulus $(2: 1)$. Most interesting is what occurred in the transition between 1:1 and 2:1 regions: sustained reentry could be induced with a low threshold. A similar transition occurs between 2:1 and $3: 1$ behavior, although it is restricted to a narrow range of intervals.
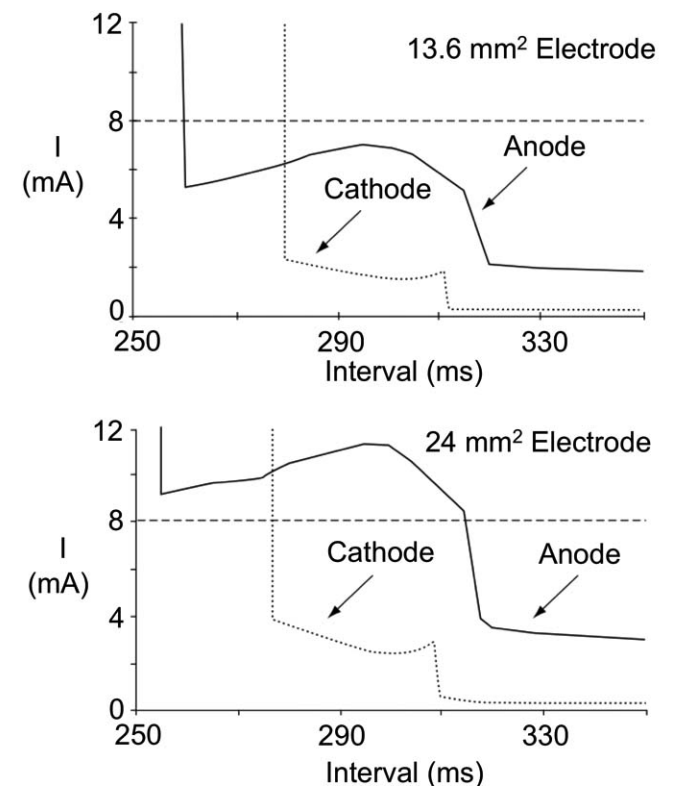

Fig. (10). The cathodal and anodal strength-interval curves, for: upper panel, a small electrode, and lower panel, a large electrode. The dashed line indicates the peak output of an electrical stimulator $(8 \mathrm{~mA})$. For the small electrode, the entire break section of the anodal curve lies below $8 \mathrm{~mA}$, and for the large electrode the break section lies above $8 \mathrm{~mA}$. Modified from [93].

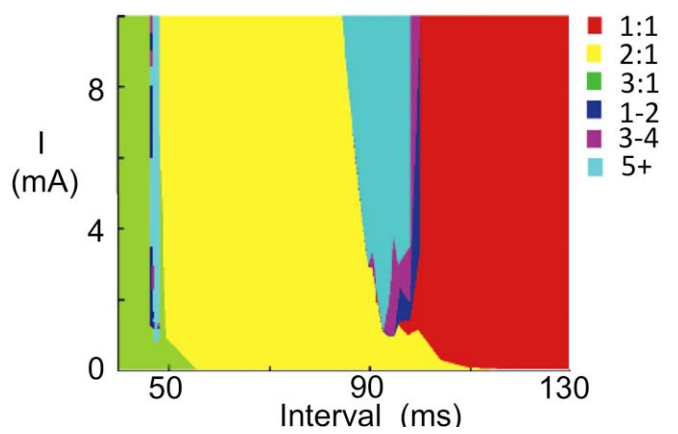

Fig. (11). The strength-interval response for cathodal unipolar pulse trains with different inter-pulse intervals. In the red region, the tissue responded 1:1 with the stimulus, in yellow $2: 1$, and in green $3: 1$. The blue, purple, and cyan regions indicate that reentry was generated by the pulse train, with varying number of rotations of the reentrant loop (blue, 1-2; purple 3-4, and cyan 5 or more). Modified from [96].

Examination of the mechanism leading to an arrhythmia suggests that burst pacing induces quatrefoil reentry, consist- 
ing of two back-to-back figure of eight reentries. Quatrefoil reentry was observed first with S1-S2 excitation, but at a much higher threshold [97], and was also predicted by bidomain simulations $[98,99]$. During S1-S2 stimulation, the key to initiating cathodal quatrefoil reentry was de-excitation of the virtual anode, creating an excitable path through which one-way propagation could occur. During burst pacing, the stimuli are far too weak to de-excite the tissue. However, apparently very small modulations of excitability can have a significant impact if delivered when the tissue is just barely able to support excitation (for example, when it is just about to transition between $1: 1$ and $2: 1$ excitation). In this case, initiation of reentry is extremely sensitive to the precise number of pulses in the pulse train.

\section{CONCLUSION}

The bidomain model has proven to be an excellent tool for understanding cardiac pacing. It has resulted in a series of theoretical predictions that have been confirmed experimentally, usually using optical mapping. These studies have clarified a number of long-standing questions, such as the mechanisms of make and break excitation, and the dip in the anodal strength-interval curve. The success of the bidomain model suggests that it may also be useful for modeling the response of the heart to strong shocks during defibrillation. Indeed, another review at least as long as this one could be written describing the use of the bidomain model for studying defibrillation [100, 101].

\section{REFERENCES}

[1] Beck H, Boden WE, Patibandla S, et al. $50^{\text {th }}$ anniversary of the first successful permanent pacemaker implantation in the United States: Historical review and future directions. Amer J Cariol 2010; 106: $810-18$.

[2] Jeffrey K. Machines in Our Hearts: The Cardiac Pacemaker, the Implantable Defibrillator,and American Health Care. Baltimore, MD: Johns Hopkins University Press; 2001.

[3] Janks D, Roth BJ. The bidomain theory of pacing. In: Cardiac Bioelectric Therapy: Mechanisms and Practical Implications. Efimov IR, Kroll M, Tchou P, Eds, Springer, New York, 63-83, 2009.

[4] Wikswo JP Jr, Roth BJ. Virtual electrode theory of pacing. In: Cardiac Bioelectric Therapy: Mechanisms and Practical Implications. Efimov IR, Kroll M, Tchou P, Eds, Springer, New York, 283-330, 2009.

[5] Efimov IR, Gray RA, Roth BJ. Virtual electrodes and deexcitation: new insights into fibrillation induction and defibrillation. J Cardiovasc Electrophysiol 2000; 11: 339-53.

[6] Henriquez CS. Simulating the electrical behavior of cardiac tissue using the bidomain model. Crit Rev Biomed Eng 1993; 21: 1-77.

[7] Neu JC, Krassowska W. Homogenization of syncytial tissues. Crit Rev Biomed Eng 1993; 21: 137-199.

[8] Roth BJ. Bidomain model. Scholarpedia http://www.scholarpedia. org/article/Bidomain_model.

[9] Muler A, Markin $\bar{V}$. Electrical properties of anisotropic nerve muscle syncytia: I. Distribution of the electrotonic potential. Biofizika 1977; 22: 307-12.

[10] Miller WT III, Geselowitz DB. Simulation studies of the electrocardiogram: I. The normal heart. Circ Res 1978; 43: 301-15.

[11] Tung L. A bidomain model for describing ischemic myocardial dc Potentials. PhD dissertation, Massachusetts Inst Technol, Cambridge, 1978.

[12] Peskoff A. Electric potential in three-dimensional electrically syncytial tissues. Bull Math Biol 1979; 41: 163-81.

[13] Eisenberg RS, Barcilon V, Mathias RT. Electrical properties of spherical syncytia. Biophys J 1979; 25: 151-80.

[14] Geselowitz DB, Miller WT III. A bidomain model for anisotropic cardiac muscle. Ann Biomed Eng 1983; 11: 191-206.
[15] Plonsey R, Barr RC. Current flow patterns in two-dimensional anisotropic bisyncytia with normal and extreme conductivities. Biophys J 1984; 45: 557-71.

[16] Barr RC, Plonsey R. Propagation of excitation in idealized anisotropic two-dimensional tissue. Biophys J 1984; 45: 1191-202.

[17] Roth BJ, WIkswo JP Jr. A bidomain model for the extracellular potential and magnetic field of cardiac tissue. IEEE Trans Biomed Eng 1986; 33: 467-9.

[18] Sepulveda NG, Wikswo JP Jr. Electric and magnetic fields from two-dimensional bisyncytia. Biophys J 1987; 51: 557-68.

[19] Plonsey R, Barr RC. Interstitial potentials and their change with depth into cardiac tissue. Biophys J 1987; 51: 547-55.

[20] Sepulveda NG, Roth BJ, Wikswo JP Jr. Current injection into a two-dimensional anisotropic bidomain. Biophys J 1989; 55: 987-99.

[21] Mounsey JP, Knisley SB. Anodal capture, cathodal capture, and left ventricular cardiac excitation. J Cardiovasc Electrophysiol 2009; 20: 650-2.

[22] Thakor N, Ranjan R, Rajasekhar S, Mower M. Effect of varying pacing waveform shapes on propagation of hemodynamics in the rabbit heart. Am J Cardiol 1997; 79: 36-43.

[23] Thakral A, Stein LH, Shenai M, Gramatikov B, Thakor N. Effects of anodal vs cathodal pacing on the mechanical performance of the isolated rabbit heart. J Appl Physiol 2000; 89: 1159-64.

[24] Theis C, Bavikati VV, Langberg JJ, Lloyd MS. The relationship of bipolar left ventricular pacing stimulus intensity to cardiac depolarization and repolarization in humans with cardiac resynchronization devices. J Cardiovasc Electrophysiol 2009; 20: 645-9.

[25] Roth BJ, Wikswo JP Jr. Electrical stimulation of cardiac tissue: A bidomain model with active membrane properties. IEEE Trans Biomed Eng 1994; 41: 232-40.

[26] Muzikant AL, Henriquez CS. Paced activation mapping reveals organization of myocardial fibers. J Cardiovasc Electrophysiol 1997; 8: 281-94.

[27] Muzikant AL, Henriquez CS. Bipolar stimulation of a threedimensional bidomain incorporating rotational anisotropy. IEEE Trans Biomed Eng 1998; 45: 449-62.

[28] Muzikant AL, Henriquez CS. Validation of three-dimensional conduction models using experimental mapping: Are we getting closer? Prog Biophys Mol Biol 1998; 69: 205-23.

[29] Suzuki T, Sato S, Ohe T, Susuki R, Kajiya F. Analysis of the virtual electrode phenomena using bidomain model: Basic characteristics for passive membrane. J Mech Med Biol 2006; 6: 455-75.

[30] Patel SG, Roth BJ. How electrode size affects the electric potential distribution in cardiac tissue. IEEE Trans Biomed Eng 2000; 47: 1284-7.

[31] Goel V, Roth BJ. Approximate analytical solutions to the bidomain equations describing electrical activity in cardiac tissue. Proc $13^{\text {th }}$ Southern Biomed Eng Conf 1994; 967-70.

[32] Roth BJ. Approximate analytical solutions to the bidomain equations with unequal anisotropy ratios. Phys Rev E 1997; 55: 181926.

[33] Roth BJ. How the anisotropy of the intracellular and extracellular conductivities influences stimulation of cardiac muscle. J Math Biol 1992; 30: 633-46.

[34] Roth BJ. Electrical conductivity values used with the bidomain model of cardiac tissue. IEEE Trans Biomed Eng 1997; 44: 326-8.

[35] Roth BJ. How to explain why "unequal anisotropy ratios" is important using pictures but no mathematics. $28^{\text {th }}$ Annual International Conference of the IEEE EMBS 2006.

[36] Akar FG, Roth BJ, Rosenbaum DS. Optical measurement of cellto-cell coupling in the intact heart using subthreshold electrical stimulation. Amer J Physiol 2001; 281: H533-H542.

[37] Efimov IR, Cheng Y, Van Wagoner DR, Mazgalev T, Tchou PJ. Virtual electrode-induced phase singularity: A basic mechanism of defibrillation failure. Circ Res 1998; 82: 918-25.

[38] Skouibine KG, Trayanova NA, Moore PK. Anode/cathode make and break phenomena in a model of defibrillation. IEEE Trans Biomed Eng 1999; 46: 769-77.

[39] Trayanova N. Concepts of ventricular defibrillation. Phil Trans R Roc Lond A 2001; 359: 1327-37.

[40] Wikswo JP Jr, Wisialowski TA, Altermeier WA, Balser JR, Kopelman HA, Roden DM. Virtual cathode effects during stimulation of cardiac muscle: Two-dimensional in vivo measurements. Circ Res 1991; 68: 513-30. 
[41] Knisley SB. Transmembrane voltage changes during unipolar stimulation of rabbit ventricle. Circ Res 1995; 77: 1229-39.

[42] Neunlist M, Tung L. Spatial distribution of cardiac transmembrane potentials around an extracellular electrode: dependence on fiber orientation. Biophys J 1995; 68: 2310-22.

[43] Wikswo JP Jr, Lin S-F, Abbas RA. Virtual electrodes in cardiac tissue: a common mechanism for anodal and cathodal stimulation. Biophys J 1995; 69: 2195-210.

[44] Rosenbaum DS, Jalife J. Optical Mapping of Cardiac Excitation and Arrhythmias. Armonk, NY: Futura; 2001.

[45] Dillon SM. Optical recordings in the rabbit heart show that defibrillation strength shocks prolong the duration of depolarization and the refractory period. Circ Res 1991; 69: 842-56.

[46] Davidenko JM, Pertsov AV, Salomonsz R, Baxter W, Jalife J. Stationary and drifting spiral waves of excitation in isolated cardiac muscle. Nature 1992; 355: 349-51.

[47] Knisley SB, Hill BC. Optical recordings of the effect of electrical stimulation on action potential repolarization and the induction of reentry in two-dimensional perfused rabbit epicardium. Circulation 1993; 88: 2402-14.

[48] Roth BJ. Artifacts, assumptions, and ambiguity: Pitfalls in comparing experimental results to numerical simulations when studying electrical stimulation of the heart. Chaos 2002; 12: 973-81.

[49] Roth BJ, Pertsov AM. Hybrid modeling of electrical and optical behavior in the heart. Physica D: Nonlinear Phenomena 2009; 238: 1019-27.

[50] Latimer DC, Roth BJ. Electrical stimulation of cardiac tissue by a bipolar electrode in a conductive bath. IEEE Trans Biomed Eng 1998; 45: 1449-58.

[51] Trayanova N, Roth BJ. Mechanisms for cardiac stimulation. Proc $15^{\text {th }}$ Annu Conf IEEE EMBS 1993; 817-18.

[52] Sepulveda NG, Wikswo JP. Bipolar stimulation of cardiac tissue using an anisotropic bidomain model. J Cardiovasc Electrophysiol 1994; 5: 258-67.

[53] Roth BJ. A comparison of two boundary conditions used with the bidomain model of cardiac tissue. Ann Biomed Eng 1991; 19: 66978.

[54] Krassowska W, Neu JC. Effective boundary conditions for syncytial tissues. IEEE Trans Biomed Eng 1994; 41: 143-50.

[55] Constantino J, Long Y, Ashihara T, Trayanova N. Tunnel propagation following defibrillation with ICD shocks: hidden postshock activations in the left ventricular wall underlie isoelectric window. Heart Rhythm 2010; 7: 953-61.

[56] Gray RA. What exactly are optically recorded "action potentials." J Cardiovasc Electrophysiol 1999; 10: 1463-6.

[57] Girouard SD, Laurita KR, Rosenbaum DS. Unique properties of cardiac action potentials recorded with voltage-sensitive dyes. J Cardiovasc Electrophysiol 1996; 7: 1024-38.

[58] Al-Khadra A, Nikolski V, Efimov IR. The role of electroporation in defibrillation. Circ Res 2000; 87: 797-804.

[59] Baxter WT, Mironov SF, Zaitsev AV, Jalife J, Pertsov AM. Visualizing excitation waves inside cardiac muscle using transillumination. Biophys J 2001; 80: 516-30.

[60] Janks D, Roth BJ. Averaging over depth during optical mapping of unipolar stimulation. IEEE Trans Biomed Eng 2002; 49: 1051-4.

[61] Prior P, Roth BJ. Calculation of optical signal using threedimensional bidomain/diffusion model reveals distortion of the transmembrane potential. Biophys J 2008; 95: 2097-102.

[62] Poelzing S, Roth BJ, Rosenbaum DS. Optical measurements reveal nature of intercellular coupling across the ventricular wall. Am J Physiol 2005; 289: H1428-H35.

[63] Prior PW, Roth BJ. Development of an imaging modality utilizing 2-D optical signals during an epi-flourescent optical mapping experiment. Phys Med Biol 2009; 54: 3015-030.

[64] Walton RD, Benoist D, Hyatt C, Gilbert SH, White E, Bernus O. Dual excitation wavelength epifluorescence imaging of transmural electrophysiological properties in intact hearts. Heart Rhythm 2010; 7: 1843-9.

[65] Neunlist M, Zou SZ, Tung L. Design and use of an "optrode" for optical recordings of cardiac action potentials. Pflugers Arch 1992; 420: 611-17.

[66] Byars JL, Smith WM, Ideker RE, Fast VG. Development of an optrode for intramural multisite optical recordings of $V \mathrm{~m}$ in the heart. J Cardiovasc Electrophysiol 2003; 14: 1196 -202.

[67] Caldwell BJ, LeGrice IJ, Hooks DA, Tai DC, Pullan AJ, Smaill $\mathrm{BH}$. Intramural measurement of transmembrane potential in the isolated pig heart: validation of a novel technique. J Cardiovasc Electrophysiol 2005; 16: 1001-10.

[68] Sharifov OF, Fast VG. Intramural virtual electrodes in ventricular wall effects on epicardial polarizations. Circulation 2004; 109: 2349-56.

[69] Goto M, Brooks C McC. Membrane excitability of the frog ventricle examined by long pulses. Amer J Physiol 1969; 217: 1236-45.

[70] Dekker E. Direct current make and break thresholds for pacemaker electrodes on the canine ventricle. Circ Res 1970; 27: 811-23.

[71] Ehara T. Rectifier properties of canine papillary muscle. Jpn J Physiol. 1971; 21: 49-69.

[72] Lindemans FW, Heetharr RM, Denier Van der Gon JJ, Zimmerman ANE. Site of initial excitation and current threshold as a function of electrode radius in heart muscle. Cardiovasc Res 1975; 9: 95-104.

[73] Roth BJ. A mathematical model of make and break electrical stimulation of cardiac tissue by a unipolar anode or cathode. IEEE Trans Biomed Eng 1995; 42: 1174-84.

[74] Hodgkin AL, Huxley AF. A quantitative description of membrane current and its application to conduction and excitation in nerve. $\mathbf{J}$ Physiol 1952; 117: 500-44.

[75] Ranjan R, Chiamvimonvat N, Thakor NV, Tomaselli GF, Marban E. Mechanism of anode break stimulation in the heart. Biophys $\mathrm{J}$ 1998; 74: 1850-63.

[76] Ranjan R, Tomaselli GF, Marban E. A novel mechanism of anodebreak stimulation predicted by bidomain modeling. Circ Res 1999; 84: 153-6.

[77] Roth BJ, Chen J. Mechanism of anode break excitation in the heart: The relative influence of membrane and electrotonic factors. J Biol Systems 1999; 7: 541-52.

[78] Colli-Franzone P, Pavarino LF, Scacchi S. Exploring anodal and cathodal make and break excitation in a 3D anisotropic bidomain model. Math Biosci 2011; 230: 96-114.

[79] Nikolski V, Sambelashvili A, Efimov IR. Anode-break excitation during end-diastolic stimulation is explained by half-cell double layer discharge. IEEE Trans Biomed Eng 2002; 49: 1217-20.

[80] Nikolski VP, Sambelashvili AT, Efimov IR. Mechanisms of make and break excitation revisited: Paradoxical break excitation during diastolic stimulation. Amer J Physiol 2002; 282: H565-H75.

[81] Sambelashvili AT, Nikolski VP, Efimov IR. Nonlinear effects in subthreshold virtual electrode polarization. Am J Physiol 2003; 284: H2368-H74.

[82] van Dam RTh, Durrer D, Strackee J, van der Twell LH. The excitability cycle of the dog's left ventricle determined by anodal, cathodal and bipolar stimulation. Circ Res 1956; 4: 196-204.

[83] Cranefield PF, Hoffman BF, Siebens AA. Anodal excitation of cardiac muscle. Am J Physiol 1957; 190: 383-90.

[84] Mehra R, Furman S. Comparison of cathodal, anodal, and bipolar strength-interval curves with temporary and permanent pacing electrodes. Brit Heart J 1979; 41: 468-76.

[85] Roth BJ. Strength-interval curves for cardiac tissue predicted using the bidomain model. J Cardiovasc Electrophysiol 1996; 7: 722-37.

[86] Sidorov VY, Woods MC, Baudenbacher P, Baudenbacher F. Examination of stimulation mechanism and strength-interval curve in cardiac tissue. Am J Physiol Heart 2005; 289: H2602-15.

[87] Sidorov VY, Woods MC, Wikswo JP. Effects of elevated extracellular potassium on the stimulation mechanism of diastolic cardiac tissue. Biophys J 2003; 84: 3470-9.

[88] Roth BJ, Patel SG. Effects of elevated extracellular potassium ion concentration on anodal excitation of cardiac tissue. J Cardiovasc Electrophysiol 2003; 14: 1351-5.

[89] Joung BY, Park HW, Maruyama M, et al. Intracellular calcium and the mechanism of anodal supernormal excitability in Langendorff perfused rabbit ventricles. Circ J 2011; 75: 834-43.

[90] Sidorov VY, Holcomb MR, Woods MC, Gray RA, Wikswo JP. Effects of unipolar stimulation on voltage and calcium distributions in the isolated rabbit heart. Basic Res Cardiol 2008; 103: 537-51.

[91] Hayashi H, Lin S-F, Joung B, Karagueuzian H, Weiss JN, Chen P-S. Virtual electrodes and the induction of fibrillation in langendorffperfused rabbit ventricles: The role of intracellular calcium. Am J Physiol 2008; 295: H1422-H8.

[92] Mehra R, McMullen M, Furman S. Time-dependence of unipolar cathodal and anodal strength-interval curves. PACE 1980; 3: 526-30.

[93] Bennett JA, Roth BJ. Time dependence of anodal and cathodal refractory periods in cardiac tissue. PACE 1999; 22: 1031-8. 
[94] El-Sherif N, Gough WB, Restivo M. Reentrant ventricular arrhythmias in the late myocardial infarction period: 14. Mechanisms of resetting, entrainment, acceleration, or termination of reentrant tachycardia by programmed electrical stimulation. PACE 1987; 10: 341-71.

[95] El-Sherif N, Mehra R, Gough WB, Zeiler RH. Reentrant ventricular arrhythmias in the late myocardial infarction period: II. Burst pacing versus multiple premature stimulation in the induction of reentry. J Am Coll Cardiol 1984; 4: 295-304.

[96] Janks DL, Roth BJ. Quatrefoil reentry caused by burst pacing. J Cardiovasc Electrophysiol 2006; 17: 1362-8.

[97] Lin S-F, Roth BJ, Wikswo JP Jr. Quatrefoil reentry in myocardium: An optical imaging study of the induction mechanism. J Cardiovasc Electrophysiol 1999; 10: 574-86.
[98] Saypol JM, Roth BJ. A mechanism for anisotropic reentry in electrically active tissue. J Cardiovasc Electrophysiol 1992; 3: 558-66.

[99] Roth BJ. Nonsustained reentry following successive stimulation of cardiac tissue through a unipolar electrode. J Cardiovasc Electrophysiol 1997; 8: 768-78.

[100] Trayanova N, Plank G. Bidomain model of defibrillation. In: Cardiac Bioelectric Therapy: Mechanisms and Practical Implications. Efimov IR, Kroll M, Tchou P, Eds, Springer, New York, 85-109, 2009.

[101] Ripplinger CM, Efimov IR. The virtual electrode hypothesis of defibrillation. In: Cardiac Bioelectric Therapy: Mechanisms and Practical Implications. Efimov IR, Kroll M, Tchou P, Eds, Springer, New York, 331-56, 2009.

(C) Bradley J. Roth; Licensee Bentham Open.

This is an open access article licensed under the terms of the Creative Commons Attribution Non-Commercial License (http://creativecommons.org/licenses/ by-nc/3.0/) which permits unrestricted, non-commercial use, distribution and reproduction in any medium, provided the work is properly cited. 